\title{
INEQUALITIES FOR THE DERIVATIVE OF A POLYNOMIAL
}

\author{
ABDUL AZIZ
}

\begin{abstract}
Let $P(z)=\sum_{j=0}^{n} a_{j} z^{j}=c \prod_{j=1}^{n}\left(z-z_{j}\right)$ be a polynomial of degree $n$ and $P^{\prime}(z)$ its derivative. In this paper we consider the problem of estimating the maximum of $\left|P^{\prime}(z)\right|$ on $|z|=1$ under some assumptions on the zeros or on the coefficients of $P(z)$ and obtain certain sharp results.
\end{abstract}

1. Introduction and statement of results. If $P(z)$ is a polynomial of degree $n$, then concerning the estimate of $\left|P^{\prime}(z)\right|$ on the unit disk $|z| \leqslant 1$, we have the following famous result known as Bernstein's inequality (for reference see [8]):

$$
\operatorname{Max}_{|z|=1}\left|P^{\prime}(z)\right| \leqslant n \operatorname{Max}_{|z|=1}|P(z)| \text {. }
$$

In (1) equality holds if and only if $P(z)$ has all its zeros at the origin.

It was shown by Turán [10] that if $P(z)$ has all its zeros in $|z| \leqslant 1$, then

$$
\operatorname{Max}_{|z|=1}\left|P^{\prime}(z)\right| \geqslant \frac{n}{2} \underset{|z|=1}{\operatorname{Max}}|P(z)| \text {. }
$$

On the other hand, P. Erdös conjectured, and later Lax [5] proved, that if $P(z) \neq 0$ in $|z|<1$, then

$$
\operatorname{Max}_{|z|=1}\left|P^{\prime}(z)\right| \leqslant \frac{n}{2} \underset{|z|=1}{\operatorname{Max}}|P(z)|
$$

Hence, in (2) as well as in (3), equality holds for those polynomials of degree $n$ which have all their zeros on $|z|=1$. As an extension of (2), Govil [3] proved that if $P(z)$ has all its zeros in $|z| \leqslant K$ where $K \geqslant 1$, then

$$
\operatorname{Max}_{|z|=1}\left|P^{\prime}(z)\right| \geqslant \frac{n}{1+K^{n}} \underset{|z|=1}{\operatorname{Max}}|P(z)|,
$$

whereas if $P(z) \neq 0$ in $|z|<K, K \geqslant 1$, then [6]

$$
\operatorname{Max}_{|z|=1}\left|P^{\prime}(z)\right| \leqslant \frac{n}{1+K} \underset{|z|=1}{\operatorname{Max}}|P(z)| \text {. }
$$

In [2] Giroux, Rahman and Schmeisser have mentioned the following problem which they have attributed to E. B. Saff.

Received by the editors March 21, 1981.

1980 Mathematics Subject Classification. Primary 30A06, 30A40; Secondary 26A82, 30A04.

Key words and phrases. Derivative of a polynomial, inequalities in the complex domain, self-inversive polynomials. 
Problem 1. Let $P(z)=\prod_{j=1}^{n}\left(z-z_{j}\right)$ be a polynomial of degree $n$ having all its zeros in $\operatorname{Re} z \geqslant 1$. Is it true that

$$
\operatorname{Max}_{|z|=1}\left|P^{\prime}(z)\right| \leqslant \sum_{j=1}^{n} \frac{1}{1+\operatorname{Re} z_{j}} \underset{|z|=1}{\operatorname{Max}}|P(z)| ?
$$

In full generality (6) is proved (see [2, Theorem 1]) only for polynomials of degree $\leqslant 2$. If the polynomial $P(z)$ of the above problem is such that $\left|z_{j}\right| \leqslant 1, j=1,2, \ldots, n$, then [2, Theorem 5]

$$
\operatorname{Max}_{|z|=1}\left|P^{\prime}(z)\right| \geqslant \sum_{j=1}^{n} \frac{1}{\left(1+\left|z_{j}\right|\right)} \operatorname{Max}_{|z| \neq 1}|P(z)| .
$$

In this paper we shall first obtain a generalization of (7) which is also a refinement of (4) and thereby give an independent proof of (4) as well. We prove

THEOREM 1. If all the zeros of the polynomial $P(z)=\prod_{j, 1}^{n}\left(z-z_{j}\right)$ of degree $n$ lie in $|z| \leqslant K$ where $K \geqslant 1$, then

$$
\operatorname{Max}_{|z|=1}\left|P^{\prime}(z)\right| \geqslant \frac{2}{1+K^{n}} \sum_{j=1}^{n} \frac{K}{K+\left|z_{j}\right|} \operatorname{Max}_{|z|=1}|P(z)| .
$$

The result is best possible and equality in (8) holds for $P(z)=z^{n}+K^{n}$.

If the polynomial $P(z)$ of Problem 1 is such that $|P(z)|$ and $\left|P^{\prime}(z)\right|$ become maximum at the same point on $|z|=1$, then considerably more than (6) holds true. In fact, in this case we have the following theorem which we only state here and omit its proof, since its result was known to those who have worked on the Saff conjecture'.

THEOREM 2. If all the zeros of the polynomial $P(z)=\prod_{j=1}^{n}\left(z-z_{j}\right)$ of degree $n$ lie in $|z| \geqslant 1$, then

$$
\operatorname{Max}_{|z|=1}\left|P^{\prime}(z)\right| \leqslant \sum_{j=1}^{n} \frac{1}{1+\left|z_{j}\right|} \underset{|z|=1}{\operatorname{Max}}|P(z)|,
$$

provided $|P(z)|$ and $\left|P^{\prime}(z)\right|$ become maximum at the same point on $|z|=1$. The result is sharp.

REMARK. Let the polynomial $P(z)$ of Theorem 2 be real for real $z$. Let

$$
D_{1}=\{z \in C: \operatorname{Re} z \geqslant 0,|z| \geqslant 1\} \quad \text { and } D_{2}=\{z \in C: \operatorname{Re} z \leqslant 0,|z| \geqslant 1\} .
$$

Then $P^{\prime}(z)$ is real for real $z$ and we have

$$
\operatorname{Max}_{|z|=1}|P(z)|=|P(-1)|, \quad \operatorname{Max}_{|z|=1}\left|P^{\prime}(z)\right|=\left|P^{\prime}(-1)\right|
$$

provided all the zeros of $P(z)$ lie in $D_{1}$, and

$$
\underset{|z|=1}{\operatorname{Max}}|P(z)|=|P(1)|, \quad \operatorname{Max}_{|z|=1}\left|P^{\prime}(z)\right|=\left|P^{\prime}(1)\right|
$$

\footnotetext{
${ }^{1}$ This was pointed out by the referee.
} 
provided all the zeros of $P(z)$ lie in $D_{2}$. Hence, it follows from Theorem 2 that

$$
\operatorname{Max}_{|=|=1}\left|P^{\prime}(z)\right| \leqslant \sum_{j=1}^{n} \frac{1}{1+\left|z_{j}\right|} \operatorname{Max}_{k=1}|P(z)|
$$

provided all the zeros of $P(z)$ lie in $D_{1}$ or all the zeros of $P(z)$ lie in $D_{2}$. The first assertion of $(10)$ is precisely Theorem 2 of [2].

If $P(z)$ is a self-inversive polynomial of degree $n$, that is if $P(z) \equiv Q(z)$ where $Q(z)=z^{n} \overline{P(1 / \bar{z})}$, then $[9]$ (see also [1])

$$
\operatorname{Max}_{|=|=1}\left|P^{\prime}(z)\right|=\frac{n}{2} \operatorname{Max}_{|=|=1}|P(z)| \text {. }
$$

On the other hand, if $P(z)$ is a polynomial of degree $n$ satisfying $P(z) \equiv z^{n} P(1 / z)$, then the precise upper bound for the maximum of $\left|P^{\prime}(z)\right|$ on $|z|=1$ does not seem to be easily obtainable. In this connection Professor Q. I. Rahman mentioned the following problem.

Problem 2. Let $P(z)$ be a polynomial of degree $n$ satisfying $P(z) \equiv z^{n} P(1 / z)$. Is it true that

$$
\operatorname{Max}_{|=|=1}\left|P^{\prime}(z)\right| \leqslant \frac{n}{\sqrt{2}} \operatorname{Max}_{|=|=1}|P(z)| ?
$$

If $P(z)$ happens to be real for real $z$, then clearly

$$
P(z) \equiv z^{n} P(1 / z) \equiv z^{n} \overline{P(1 / \Sigma)}=Q(z)
$$

and we have, by (11),

$$
\operatorname{Max}_{|=|-1}\left|P^{\prime}(z)\right|=\frac{n}{2} \underset{|=|=1}{\operatorname{Max}}|P(z)| \text {. }
$$

We have no idea whether (12) is true or not, in general, for polynomials of degree $n \geqslant 3$ with complex coefficients. The case $n=1$ is trivial. Here we prove

THEOREM 3. If $P(z)$ is a polynomial of degree 2 satisfying $P(z) \equiv z^{n} P(1 / z)$, then

$$
\operatorname{Max}_{i=1=1}\left|P^{\prime}(z)\right| \leqslant \sqrt{2} \operatorname{Max}_{\mid=1=1}|P(z)| \text {. }
$$

The result is best possible with equality in (15) for $P(z)=z^{2}+2 i z+1$.

THEOREM 4. If $P(z)=\sum_{j=1}^{n}\left(a_{j}+i b_{j}\right) z^{j}, a_{j} \geqslant 0, b_{j} \geqslant 0, j=0,1, \ldots, n$, is a polynomial of degree $n$ with complex coefficients satisfying $P(z) \equiv z^{n} P(1 / z)$, then

$$
\operatorname{Max}_{|z|=1}\left|P^{\prime}(z)\right| \leqslant \frac{n}{\sqrt{2}} \operatorname{Max}_{|=|=1}|P(z)| \text {. }
$$

The result is best possible in the sense that the equality in (16) holds for $P(z)=z^{n}+$ $2 i z^{n / 2}+1$, where $n$ is an even positive integer.

Finally we prove an inequality in the opposite direction.

THEOREM 5. If $P(z)$ is a polynomial of degree $n$ satisfying $P(z) \equiv z^{n} P(1 / z)$, then

$$
\operatorname{Max}_{|z|=1}\left|P^{\prime}(z)\right| \geqslant \frac{n}{2} \operatorname{Max}_{|z|=1}|P(z)| \text {. }
$$

The result is sharp with equality in (17) for $P(z)=z^{n}+1$. 
2. For the proofs of these theorems we need the following lemmas.

LEMMA 1. If $P(z)$ is a polynomial of degree $n$, then on $|z|=1$,

$$
\left|P^{\prime}(z)\right|+\left|Q^{\prime}(z)\right| \leqslant n \operatorname{Max}_{|z|=1}|P(z)|,
$$

where $Q(z)=z^{n} \overline{P(1 / \bar{z})}$.

This is a special case of a result due to Govil and Rahman [4, Lemma 10].

Lemma 2. If $P(z)$ is a polynomial of degree $n$, then for $|z|=1$ and for all $R \geqslant 1$,

$$
|P(R z)-P(z)|+|Q(R z)-Q(z)| \leqslant\left(R^{n}-1\right) \underset{|z|=1}{\operatorname{Max}}|P(z)|
$$

where $Q(z)=z^{n} \overline{P(1 / \bar{z})}$.

Proof of Lemma 2. For every real or complex number $\alpha$ such that $|\alpha|=1$, we have, by Lemma 1 ,

$$
\left|P^{\prime}(z)+\alpha Q^{\prime}(z)\right| \leqslant n \operatorname{Max}_{|z|=1}|P(z)| \text { for }|z|=1 .
$$

Now, if $F(z)$ is a polynomial of degree $n$, then (see [7, Volume I, Problem 269, p. 137])

$$
\operatorname{Max}_{|z|=R>1}|F(z)| \leqslant R^{n} \operatorname{Max}_{|z|=1}|F(z)| .
$$

Applying this result to the polynomial $P^{\prime}(z)+\alpha Q^{\prime}(z)$ which is of degree $n-1$, we obtain for all $t \geqslant 1$ and $0 \leqslant \theta<2 \pi$,

$$
\left|P^{\prime}\left(t e^{i \theta}\right)+\alpha Q^{\prime}\left(t e^{i \theta}\right)\right| \leqslant n t^{n-1} \operatorname{Max}_{|z|=1}|P(z)| .
$$

Choosing argument of $\alpha$ suitably, we get

$$
\left|P^{\prime}\left(t e^{i \theta}\right)\right|+\left|Q^{\prime}\left(t e^{i \theta}\right)\right| \leqslant n t^{n-1} \operatorname{Max}_{|z|=1}|P(z)| \text { for } 0 \leqslant \theta<2 \pi \text { and } t \geqslant 1 .
$$

Also for each $\theta, 0 \leqslant \theta<2 \pi$ and $R>1$, we have

$$
P\left(R e^{i \theta}\right)-P\left(e^{i \theta}\right)=\int_{1}^{R} e^{i \theta} P^{\prime}\left(t e^{i \theta}\right) d t
$$

and

$$
Q\left(R e^{i \theta}\right)-Q\left(e^{i \theta}\right)=\int_{1}^{R} e^{i \theta} Q^{\prime}\left(t e^{i \theta}\right) d t
$$

Hence,

$$
\begin{aligned}
&\left|P\left(R e^{i \theta}\right)-P\left(e^{i \theta}\right)\right|+\mid Q\left(R e^{i \theta}\right)-Q\left(e^{i \theta}\right) \mid \leqslant \int_{1}^{R}\left(\left|P^{\prime}\left(t e^{i \theta}\right)\right|+\left|Q^{\prime}\left(t e^{i \theta}\right)\right|\right) d t \\
& \leqslant\left(\int_{1}^{R} n t^{n-1} d t\right) \operatorname{Max}_{|z|=1}|P(z)|=\left(R^{n}-1\right) \underset{|z|=1}{\operatorname{Max}}|P(z)|
\end{aligned}
$$

for $0 \leqslant \theta<2 \pi$ and $R>1$, which is equivalent to the desired result. 
We also need

LEMMA 3. If $P(z)$ is a polynomial of degree $n$ which has all its zeros in the disk $|z| \leqslant K$ where $K \geqslant 1$, then

$$
\operatorname{Max}_{|z|=K}|P(z)| \geqslant \frac{2 K^{n}}{1+K^{n}} \underset{|z|=1}{\operatorname{Max}}|P(z)| \text {. }
$$

The result is best possible and equality in (20) holds for $P(z)=z^{n}+K^{n}$.

Proof of Lemma 3. Since $P(z)$ has all its zeros in the disk $|z| \leqslant K, K \geqslant 1$, we write

$$
P(z)=c \prod_{j=1}^{n}\left(z-r_{j} e^{i \theta_{j}}\right) \quad \text { where } r_{j} \leqslant K, j=1,2, \ldots, n .
$$

Then, clearly, for points $e^{i \theta}, 0 \leqslant \theta<2 \pi$, other than the zeros of $P(z)$, we have

This implies

$$
\begin{aligned}
\left|P\left(K^{2} e^{i \theta}\right) / P\left(e^{i \theta}\right)\right| & =\prod_{j=1}^{n}\left|\left(K^{2} e^{i \theta}-r_{j} e^{i \theta_{j}}\right) /\left(e^{i \theta}-r_{j} e^{i \theta_{j}}\right)\right| \\
& =\prod_{j=1}^{n}\left\{\frac{K^{4}+r_{j}^{2}-2 K^{2} r_{j} \cos \left(\theta-\theta_{j}\right)}{1+r_{j}^{2}-2 r_{j} \cos \left(\theta-\theta_{j}\right)}\right\}^{1 / 2} \\
& \geqslant \prod_{j=1}^{n} K=K^{n} .
\end{aligned}
$$

$$
\left|P\left(K^{2} e^{i \theta}\right)\right| \geqslant K^{n}\left|P\left(e^{i \theta}\right)\right|
$$

for points $e^{i \theta}, 0 \leqslant \theta<2 \pi$, other than the zeros of $P(z)$. Since this inequality is trivial for points $e^{i \theta}$ which are the zeros of $P(z)$, it follows that

$$
\left|P\left(K^{2} z\right)\right| \geqslant K^{n}|P(z)| \text { for }|z|=1 \text {. }
$$

Let $G(z)=P(K z)$ and $H(z)=z^{n} \overline{G(1 / \bar{z})}=z^{n} \overline{P(K / \bar{z})}$. Applying Lemma 2 to the polynomial $G(z)$ we get for $|z|=1$ and for all $R \geqslant 1$

$$
|G(R z)|+|H(R z)| \leqslant\left(R^{n}+1\right) \underset{|z|=1}{\operatorname{Max}}|G(z)| .
$$

Taking, in particular, $R=K \geqslant 1$, we obtain

$$
|G(K z)|+|H(K z)| \leqslant\left(1+K^{n}\right) \underset{|z|=1}{\operatorname{Max}}|G(z)| \quad \text { for }|z|=1 .
$$

Equivalently,

$$
\left|P\left(K^{2} z\right)\right|+K^{n}|P(z)| \leqslant\left(1+K^{n}\right) \underset{|z|=K}{\operatorname{Max}}|P(z)| \text { for }|z|=1 .
$$

This gives, with the help of (21),

$$
2 K^{n}|P(z)| \leqslant\left(1+K^{n}\right) \underset{|z|=K}{\operatorname{Max}}|P(z)| \text { for }|z|=1 .
$$

Hence,

$$
\operatorname{Max}_{|z|=K}|P(z)| \geqslant \frac{2 K^{n}}{1+K^{n}} \underset{|z|=1}{\operatorname{Max}}|P(z)| .
$$

This proves the desired result. 
For the proof of Theorem 5 we need

Lemma 4. Let $P(z)$ be a polynomial of degree $m$. If for some positive integer $n \geqslant m$, $P(z) \equiv z^{n} \overline{P(1 / \bar{z})}$, then

$$
\operatorname{Max}_{|z|=1}\left|P^{\prime}(z)\right| \leqslant \frac{n}{2} \operatorname{Max}_{|z|=1}|P(z)| \text {. }
$$

The result is best possible with equality in (22) for $P(z)=z^{m}+z^{n-m}, m \leqslant n \leqslant 2 m$.

Proof of Lemma 4. If $m=n$, then $P(z)$ is a self-inversive polynomial of degree $n$ and the result follows from (12). So we suppose that $m<n$. We take $F(z)=\alpha z^{n}+$ $\bar{\alpha}+P(z)$ where $\alpha$ is a complex number such that $|\alpha|=1$. Then

$$
z^{n} \overline{F(1 / \bar{z})}=\bar{\alpha}+z^{n} \alpha+z^{n} \overline{P(1 / \bar{z})} \equiv \alpha z^{n}+\bar{\alpha}+P(z)=F(z)
$$

This shows that $F(z)$ is a self-inversive polynomial of degree $n$ and, therefore, it follows from (11) that

$$
\left|F^{\prime}(z)\right| \leqslant \frac{n}{2} \operatorname{Max}_{|z|=1}|F(z)| \text { for }|z|=1 .
$$

Equivalently,

$$
\begin{aligned}
& \left|n \alpha z^{n-1}+P^{\prime}(z)\right| \leqslant \frac{n}{2} \operatorname{Max}_{|z|=1}\left|\alpha z^{n}+\bar{\alpha}+P(z)\right| \\
& \leqslant \frac{n}{2}\left(2+\operatorname{Max}_{|z|=1}|P(z)|\right) \quad \text { for }|z|=1 .
\end{aligned}
$$

Choosing argument of $\alpha$ suitably we obtain

$$
n+\left|P^{\prime}(z)\right| \leqslant n+\frac{n}{2} \underset{|z|=1}{\operatorname{Max}}|P(z)| \text { for }|z|=1 .
$$

This implies

$$
\operatorname{Max}_{|z|=1}\left|P^{\prime}(z)\right| \leqslant \frac{n}{2} \operatorname{Max}_{|z|=1}|P(z)|,
$$

which is the desired result.

\section{Proofs of the theorems.}

Proof of Theorem 1. The polynomial $G(z)=P(K z)=\prod_{j=1}^{n}\left(K z-z_{j}\right)$ has all its zeros in $|z| \leqslant 1$ and we have

$$
\frac{G^{\prime}(z)}{G(z)}=\sum_{j=1}^{n} \frac{1}{z-\left(z_{j} / K\right)}
$$

so that for $0 \leqslant \theta<2 \pi$

$$
\left|\frac{G^{\prime}\left(e^{i \theta}\right)}{G\left(e^{i \theta}\right)}\right| \geqslant \operatorname{Re} \frac{e^{i \theta} G^{\prime}\left(e^{i \theta}\right)}{G\left(e^{i \theta}\right)}=\sum_{j=1}^{n} \operatorname{Re} \frac{e^{i \theta}}{e^{i \theta}-\left(z_{j} / K\right)} \geqslant \sum_{j=1}^{n} \frac{K}{K+\left|z_{j}\right|} .
$$

This implies

$$
\operatorname{Max}_{|z|=1}\left|G^{\prime}(z)\right| \geqslant \sum_{j=1}^{n} \frac{K}{K+\left|z_{j}\right|} \operatorname{Max}_{|z|=1}|G(z)|
$$


Equivalently,

$$
K \operatorname{Max}_{|z|=1}\left|P^{\prime}(K z)\right| \geqslant \sum_{j=1}^{n} \frac{K}{K+\left|z_{j}\right|} \operatorname{Max}_{|z|=1}|P(K z)| .
$$

Since $P^{\prime}(z)$ is a polynomial of degree $n-1$, it follows by (19) that

$$
K^{n-1} \operatorname{Max}_{|z|=1}\left|P^{\prime}(z)\right| \geqslant \operatorname{Max}_{|z|=1}\left|P^{\prime}(K z)\right| .
$$

Using (24) and Lemma 3 in (23), we obtain

$$
K^{n} \operatorname{Max}_{|z|=1}\left|P^{\prime}(z)\right| \geqslant\left(\sum_{j=1}^{n} \frac{K}{K+\left|z_{j}\right|}\right) \frac{2 K^{n}}{1+K^{n}} \underset{|z|=1}{\operatorname{Max}}|P(z)|,
$$

and hence the desired result follows.

Proof of Theorem 3. The proof is simple and we leave it to the reader.

Proof of Theorem 4. We write $P(z)=P_{1}(z)+i P_{2}(z)$ where

$$
P_{1}(z)=\sum_{j=1}^{n} a_{j} z^{j}, \quad P_{2}(z)=\sum_{j=1}^{n} b_{j} z^{j}
$$

are polynomials of degree $\leqslant n$ with nonnegative coefficients. Since $P(z) \equiv z^{n} P(1 / z)$, it follows that

$$
P_{1}(z) \equiv z^{n} P_{1}(1 / z) \equiv z^{n} \overline{P_{1}(1 / \bar{z})} \quad \text { and } \quad P_{2}(z) \equiv z^{n} P_{2}(1 / z) \equiv z^{n} \overline{P_{2}(1 / \bar{z})} \text {. }
$$

Applying Lemma 4 to the polynomials $P_{1}(z)$ and $P_{2}(z)$, we obtain

$$
\operatorname{Max}_{|z|=1}\left|P_{1}^{\prime}(z)\right| \leqslant \frac{n}{2} \underset{|z|=1}{\operatorname{Max}}\left|P_{1}(z)\right|=\frac{n}{2} P_{1}(1)
$$

and

$$
\operatorname{Max}_{|z|=1}\left|P_{2}^{\prime}(z)\right| \leqslant \frac{n}{2} \underset{|z|=1}{\operatorname{Max}}\left|P_{2}(z)\right|=\frac{n}{2} P_{2}(1) .
$$

Let $\left|P^{\prime}(z)\right|$ become maximum at $z=e^{i \alpha}, 0 \leqslant \alpha<2 \pi$, on $|z|=1$, then we have

$$
\begin{aligned}
\operatorname{Max}_{|z|=1}\left|P^{\prime}(z)\right| & =\left|P^{\prime}\left(e^{i \alpha}\right)\right| \leqslant\left|P_{1}^{\prime}\left(e^{i \alpha}\right)\right|+\left|P_{2}^{\prime}\left(e^{i \alpha}\right)\right| \\
& \leqslant \frac{n}{2}\left(P_{1}(1)+P_{2}(1)\right) \leqslant \frac{n}{2} \sqrt{2\left(P_{1}^{2}(1)+P_{2}^{2}(1)\right)} \\
& =\frac{n}{\sqrt{2}}|P(1)| \leqslant \frac{n}{\sqrt{2}} \operatorname{Max}_{|z|=1}|P(z)|,
\end{aligned}
$$

and this completes the proof of Theorem 4.

Proof of Theorem 5. We have $P(z) \equiv z^{n} P(1 / z)$, therefore,

$$
z^{n-1} P^{\prime}(1 / z)=n P(z)-z P^{\prime}(z) \text {. }
$$

Now for $|z|=1$,

$$
\left|P^{\prime}(z)\right|+\left|n P(z)-z P^{\prime}(z)\right| \geqslant n|P(z)|
$$

This implies

$$
\operatorname{Max}_{|z|=1}\left|P^{\prime}(z)\right|+\underset{|z|=1}{\operatorname{Max}}\left|n P(z)-z P^{\prime}(z)\right| \geqslant n \operatorname{Max}_{|z|=1}|P(z)| .
$$


Since by (25)

$$
\underset{|z|=1}{\operatorname{Max}}\left|n P(z)-z P^{\prime}(z)\right|=\underset{|z|=1}{\operatorname{Max}}\left|z^{n-1} P^{\prime}(1 / z)\right|=\underset{|z|=1}{\operatorname{Max}}\left|P^{\prime}(z)\right|,
$$

it follows from (26) that

$$
2 \underset{|z|=1}{\operatorname{Max}}\left|P^{\prime}(z)\right| \geqslant n \operatorname{Max}_{|z|=1}|P(z)|,
$$

which is equivalent to the desired result.

\section{REFERENCES}

1. Abdul Aziz and Q. G. Mohammad, Simple proof of a Theorem of Erdös and Lax, Proc. Amer. Math. Soc. 80 (1980), 119-122.

2. A. Giroux, Q. I. Rahman and G. Schmeisser, On Bernstein's inequality, Canad. J. Math. 31 (1979), 347-353.

3. N. K. Govil, On the derivative of a polynomial, Proc. Amer. Math. Soc. 41 (1973), 543-546.

4. N. K. Govil and Q. I. Rahman, Functions of exponential type not vanishing in a half-plane and related polynomials, Trans. Amer. Math. Soc. 137 (1969), 501-517.

5. P. D. Lax, Proof of a conjecture of P. Erdös on the derivative of a polynomial, Bull. Amer. Math. Soc. 50 (1944), 509-513.

6. M. A. Malik, On the derivative of a polynomial, J. London Math. Soc. 1 (1969), 57-60.

7. G. Pólyá and G. Szegö, A ufgaben und Lehrsätze aus der Analysis, Springer-Verlag, Berlin, 1925.

8. A. C. Schaeffer, Inequalities of A. Markoff and S. Bernstein for polynomials and related functions, Bull. Amer. Math. Soc. 47 (1941), 565-579.

9. E. B. Saff and T. Sheil-Small, Coefficient and integral mean estimates for algebraic and trigonometric polynomials with restricted zeros, J. London Math. Soc. (2) 9 (1974), 16-22.

10. P. Turán, Üher die ableitung von polynomen, Compositio Math. 7 (1939), 89-95.

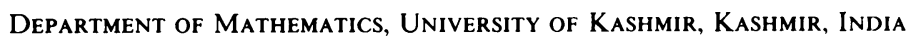

